\title{
ARTíCULO \\ Efecto de dietas en base a microalgas tradicionales, nativas y dietas artificiales sobre el crecimiento y supervivencia en larvas velígeras de la almeja taquilla, Mulinia edulis
}

\author{
Effect of traditional microalgae-based food, native and artificial diets on growth and \\ survival of the taquilla clam veliger larvae, Mulinia edulis
}

\begin{abstract}
Giovanni Vivanco' ${ }^{1}$, Doris Oliva1 ${ }^{1}$ y Alejandro Abarca ${ }^{2}$
${ }^{1}$ Instituto de Biología, Centro de Investigación y Gestión de los Recursos Naturales (CIGREN), Facultad de Ciencias, Universidad de Valparaíso, Gran Bretaña 1111, Playa Ancha, Valparaíso. doris.oliva@uv.cl

${ }^{2}$ Programa de Magíster en Acuicultura, Facultad de Ciencias del Mar, Universidad Católica del Norte, Larrondo 1281, Coquimbo, Chile
\end{abstract}

\begin{abstract}
Three sequential experiments were performed to assess the effect of 9 diets of traditionally used microalgae, native microalgae and artificial diets on the survival and growth rate of veliger larvae of the clam Mulinia edulis. Larvae were obtained from broodstock collected from natural banks in the Isla Grande de Chiloé, Región de Los Lagos, Chile. Diets were administrated as monospecific and mixed treatments. For traditional diets we used Isochrysis galbana (clone T) and Chaetoceros calcitrans; the mixed treatment (I.galbana/C.calcitrans) produced the greatest survival (13.6\%) and daily growth rate $\left(10.9 \mu \mathrm{m} \mathrm{day}{ }^{-1}\right)$. The native algae used were Chlamydomona sp. and the pennate diatom identified as $\mathrm{ND}_{2}$, isolated from the water column of a natural bank and the digestive tract of $M$. edulis, respectively. Both, the monospecific and mixed treatments with these 2 species produced $100 \%$ mortality. The experiment with artificial diet used a paste made from the diatom $\mathrm{ND}_{2}(\mathrm{PD})$ and a nutrient concentrate (NC) produced in a biodigestor. The treatments with $\mathrm{NC}$ and $\mathrm{NC} /$ PD produced $100 \%$ mortality, while with PD alone survival was $0.31 \%$ and the growth rate was $1.3 \mu \mathrm{m}$ day $^{-1}$. The best results were obtained with a diet of the traditionally used microalgae; however, the effects of native microalgae and artificial diets should also be tested in the following stages of $M$. edulis, the post-larvae and seeds.
\end{abstract}

Key words: Larval nutrition, veliger larvae, growth, survival, Mactridae

Resumen.- Se realizaron 3 experimentos de manera secuencial para probar el efecto de 9 dietas basadas en microalgas tradicionales, microalgas nativas y dietas artificiales sobre la supervivencia y tasa de crecimiento en larvas velígeras de la almeja Mulinia edulis. Las larvas se obtuvieron de reproductores recolectados desde bancos naturales en la Isla Grande de Chiloé, Región de Los Lagos, Chile. Cada dieta fue suministrada como tratamiento monoespecífico y mixto. Para el experimento con microalgas tradicionales se utilizaron Isochrysis galbana (Clon T) y Chaetoceros calcitrans siendo el tratamiento mixto (I.galbana/C.calcitrans) la dieta con la cual se obtiene los mayores valores para la supervivencia $(13,6 \%)$ y tasa diaria de crecimiento $\left(10,9 \mu \mathrm{m}\right.$ día $\left.^{-1}\right)$. Para el experimento con microalgas nativas se utilizaron las microalgas Chlamydomona sp. y una diatomea pennada $\left(\mathrm{ND}_{2}\right)$ aisladas desde la columna de agua de un banco natural y del tracto digestivo de M. edulis, respectivamente. Tanto los tratamientos monoespecíficos como el mixto generaron un $100 \%$ de mortalidad. Para el experimento con dietas artificiales se utilizaron una pasta de la diatomea $\mathrm{ND}_{2}(\mathrm{PD})$ y un concentrado nutritivo (CN) formulado mediante el uso de un biodigestor. Se obtuvo un $100 \%$ de mortalidad para los tratamientos $\mathrm{CN}$ y $\mathrm{CN} / \mathrm{PD}$, el tratamiento PD obtuvo una supervivencia de $0,31 \%$ y una tasa de crecimiento de $1,3 \mu \mathrm{m}$ día $^{-1}$. Los resultados obtenidos indican que los mejores resultados se obtienen con las microalgas tradicionales, sin embargo, es necesario probar el efecto de las microalgas aisladas y dietas artificiales en las siguientes etapas del ciclo de vida de $M$. edulis, postlarvas y semillas.

Palabras clave: Nutrición larval, larvas velígeras, crecimiento, supervivencia, Mactridae

\section{INTRODUCCIÓN}

La nutrición larval de moluscos bivalvos está asociada a la utilización de microalgas. La calidad nutricional del alimento suministrado es un factor crítico que determina la calidad y salud de las larvas y contribuye al éxito de las operaciones de acuicultura (Brown 2002). A partir de la década del 60 , cientos de microalgas han sido probadas 
como alimento, pero probablemente menos de 20 han tenido un uso amplio en la acuicultura (Duerr et al. 1998, Brown 2002, Marshall et al. 2010) siendo la producción de microalgas el factor limitante principal en un hatchery estimándose en un $30 \%$ del costo total de la producción de semillas (Rivero-Rodríguez et al. 2007).

Las microalgas deben poseer un número de atributos claves para ser usadas en acuicultura, como el tamaño y forma de la célula, una fácil ingestión y digestión, la composición bioquímica y la ausencia de toxinas (Brown 2002, Collantes \& Oliva en prensa).

La calidad nutricional de las microalgas depende principalmente de los constituyentes bioquímicos que las componen. Los porcentajes de proteínas, carbohidratos y principalmente de lípidos han sido ampliamente estudiados (Knauer \& Southgate 1999, Brown 2002, Helm \& Bourne 2006, Ponis et al. 2006). Dentro de los lípidos los ácidos grasos polinsaturados (PUFAs) derivados de las microalgas como el ácido docosahexanoico (DHA), ácido eicosapentaenoico (EPA) y el ácido araquidónico (AA) han sido identificados como esenciales para varias larvas y juveniles de moluscos bivalvos (Sargent et al. 1997, Hemaiswarya et al. 2011), sin embargo estos requerimientos aun no están bien establecidos (Knauer \& Southgate 1999).

La combinación de diferentes especies de microalgas provee un mejor balance nutricional y mejora el crecimiento a diferencia de una dieta compuesta solo por una especie de microalga (Lavens \& Sorgeloos 1996, Spolaore et al. 2006). Comercialmente, las especies de mayor uso en hatchery para la alimentación de larvas de bivalvos incluye los flagelados: Isochrysis galbana y Pavlova lutheri; y la diatomea Chaetoceros calcitrans (O'Connor \& Heasman 1997) las cuales cumplen con las propiedades nutricionales para ser utilizadas como alimento en acuicultura (Gouda et al. 2006, Collantes \& Oliva en prensa).

Además de las especies tradicionales de microalgas, los estudios se han enfocado en la utilización de microalgas nativas aisladas desde bancos naturales o del tracto digestivo siendo utilizadas como alimento de larvas de bivalvos (Gouda et al. 2006, Ponis et al. 2006, Collantes \& Oliva en prensa). Se han probado diversas dietas artificiales (alimento alternativo) tales como la producción de concentrados de microalgas (Brown \& Robert 2002) y la utilización de macroalgas (Uchida \& Numaguchi 1996, Cruz-Suárez et al. 2000).

Mulinia edulis (King \& Broderip, 1832), comúnmente llamada almeja 'taquilla', es una de las 6 especies de almejas comercialmente explotadas en Chile (SERNAPESCA 2011), siendo su cultivo una alternativa a la pesquería tradicional, pudiendo generar almejas bajo la talla mínima legal (baby clams) para los mercados internacionales (Abarca et al. 2012). Para el desarrollo de la tecnología de cultivo larval a nivel industrial es necesario identificar una dieta específica que cumpla con los requisitos necesarios para optimizar el crecimiento y la supervivencia. En esta experiencia se trabajó en base a la hipótesis de que dietas basadas en microalgas tradicionales, microalgas nativas o dietas artificiales, que cumplan las necesidades nutricionales específicas y que tengan cualidades (tamaño y morfología) que requieran las larvas velígeras de Mulinia edulis, producirán respuestas fisiológicas distintas verificables a través de las variaciones en las tasas de crecimiento y supervivencia.

\section{MATERIALES Y MÉTODOS}

\section{RECOLECCIÓN DE REPRODUCTORES Y DESOVE}

Los reproductores se obtuvieron desde 2 bancos naturales de $M$. edulis ubicados en la Isla Grande de Chiloé, Putemún $\left(42^{\circ} 25^{\prime} 52^{\prime \prime S} ; 7^{\circ} 45^{\prime} 0^{\prime \prime W}\right)$ y Quetalmahue (4151'23"S; 7358'14"W). Los desoves se realizaron en el hatchery de Orizon S.A. ubicado en Bahía Yal (4241'13"S; 7340’43"W) mediante inducción térmica aumentando la temperatura del agua de mar en $1^{\circ} \mathrm{C}$ cada media hora. La incubación se realizó en estanques de $1000 \mathrm{~L}$ con una densidad de 20 huevos $\mathrm{mL}^{-1}$ (Oliva et al. 2005). A las $48 \mathrm{~h}$ las larvas fueron recolectadas mediante el uso de tamices de 44,52 y $62 \mu \mathrm{m}$.

\section{DisEÑO EXPERIMENTAL}

El cultivo de larvas de $M$. edulis se realizó en recipientes con un volumen de $18 \mathrm{~L}$. Cada tratamiento contó con 3 réplicas distribuidas al azar en la sala de cultivo. Se utilizó agua de mar temperada a $15^{\circ} \mathrm{C}$, filtrada a $1 \mu \mathrm{m}$ y esterilizada con luz ultravioleta. El sistema de cultivo experimental se mantuvo con aireación constante y homogénea en todos los tratamientos y réplicas. La densidad de cultivo inicial fue de 10 larvas $\mathrm{mL}^{-1}$ (Oliva et al. 2005). El cambio de agua se realizó cada $48 \mathrm{~h}$ y las larvas fueron retenidas en tamices de distinto tamaño de malla de acuerdo al tamaño de las larvas. Los experimentos se realizaron de manera secuencial, finalizada la experiencia con microalgas tradicionales se siguió con microalgas nativas y luego con dietas artificiales. 


\section{CONTEO Y MEDICIÓN DE LAS LARVAS}

Las larvas fueron concentradas en un recipiente de $5 \mathrm{~L}$. Se extrajo una muestra de $10 \mathrm{~mL}$ y se agregó una gota de lugol para detener la actividad de las larvas. De la muestra principal se extrajo una submuestra de $1 \mathrm{~mL}$ y con la ayuda de una cámara Sedgewick-Rafter de $1 \mathrm{~mL} / 1 \mu \mathrm{L}$ se procedió a contar las larvas para estimar la cantidad por recipiente. La longitud valvar se midió usando un microscopio Nikon ${ }^{\circledR}$ Eclipse (Modelo E-300) con ocular graduado y calibrado según patrón del fabricante. Se midieron 30 larvas por cada réplica.

El cultivo larval contempló el desarrollo desde larva Dvelíger (que presenta un órgano ciliado llamado velum, el que le permite nadar y capturar su alimento) hasta el estado de pedivelíger, donde la larva comienza a asentarse, apareciendo un órgano contráctil cubierto de cilios, llamado pie, el que le permite explorar el sustrato. Se puso término a los experimentos cuando el $70 \%$ de las larvas en el cultivo estaban en estado de pedivelíger.

\section{Cultivo de microalgas}

Las microalgas, tradicionales y nativas, fueron cultivadas en medio Walne-Conway, enriquecido con metasilicato de sodio al $2 \%$, agregado en $1 \mathrm{~mL} \mathrm{~L}^{-1}$ al medio de cultivo final para diatomeas (Walne 1974). Los cultivos se escalaron hasta los $5 \mathrm{~L}$ y se mantuvieron con un fotoperiodo de 16:8 h luz:oscuridad; una intensidad de luz $60 \mu$ molphoton $\mathrm{m}^{-2} \mathrm{~s}^{-1} \mathrm{y}$ a una temperatura de la sala de cultivo de $18 \pm 2{ }^{\circ} \mathrm{C}$.

\section{Microalgas tradicionales}

Se utilizaron las microalgas Isochrysis galbana (Parke) (Clon T-Iso) (I) y Chaetoceros calcitrans (Paulsen) Takano $(C c)$ como tratamientos monoespecíficos $(25.000$ cél $\left.\mathrm{mL}^{-1}\right)$ y un tratamiento mixto $(I / C c)$ en una proporción de 1:1 (12.500 cél $\mathrm{mL}^{-1}$ para cada microalga). Las células fueron contadas mediante una Cámara de Neubauer con una profundidad de $0,1 \mathrm{~mm}$.

\section{Microalgas nativas}

Las microalgas nativas utilizadas son Chlamydomonas sp. (Chl) aislada de la columna de agua del banco de $M$. edulis ubicado en Quetalmahue y una diatomea nativa $\left(\mathrm{ND}_{2}\right)$ aislada del sistema digestivo de un reproductor de M. edulis. Como control se utilizó Isochrysis galbana (I) (Clon T-Iso).

Se consideraron 3 tratamientos monoespecíficos $(C h l$,
$\mathrm{ND}_{2}$ e $I$ ) con una concentración de 25.000 cél $\mathrm{mL}^{-1}$ y un tratamiento mixto $\left(C h l / N D_{2}\right)$ con una proporción de 1:1 (12.500 cél $\mathrm{mL}^{-1}$ por cada microalga). El control (I) tuvo una concentración de 25.000 cél $\mathrm{mL}^{-1}$. Las células fueron contadas mediante una Cámara de Neubauer con una profundidad de $0,1 \mathrm{~mm}$.

\section{Dietas artificiales}

Las dietas artificiales utilizadas fueron un concentrado nutritivo $(\mathrm{CN})$ del alga parda Macrocystis pyrifera (Linnaeus) C.Agardh y una pasta de diatomea (PD). El concentrado nutritivo se preparó según la metodología descrita por Paniagua \& Bückle (1985), usando un biodigestor para preparar el medio nutritivo. El biodigestor consiste en un recipiente con recirculación de agua, en cuyo interior se ubican conchas quebradas de moluscos de distinto tamaño que sirven como sustrato para las bacterias. Estas se encargan de degradar los trozos de la macrófita formándose así el medio nutritivo. Se determinó como dieta el uso del $\mathrm{CN}$ por la probable capacidad que tendrían las larvas de $M$. edulis de absorber y alimentarse de él tal como lo señalan Manahan (1983) y Wendt \& Johnson (2006), quienes indican que los invertebrados marinos son capaces de absorber desde el medio aminoácidos y materia orgánica disuelta, nutriéndose de ésta.

La pasta de diatomea (PD) preparada con la diatomea nativa $\left(N D_{2}\right)$ se obtuvo a través del método de filtración y se conservó a $-4^{\circ} \mathrm{C}$. Antes de ser suministrada, la pasta fue macerada y las células fueron separadas con un sonicador Elma ${ }^{\circledR}$ (Modelo Transsonic 460) para permitir que las larvas se alimentaran de ella.

Las dietas artificiales fueron suministradas como tratamientos monoespecíficos con una concentración de $5 \mathrm{~mL} \mathrm{~L}^{-1}$ para $\mathrm{CN}$, determinada mediante un experimento previo, y 0,5 g para PD determinada mediante el peso de 25.000 células de diatomeas y como tratamiento mixto $(\mathrm{CN} /$ PD) con las mismas concentraciones. El control Isochrysis galbana tuvo una concentración de 25.000 cél $\mathrm{mL}^{-1}$.

\section{Calidad nutricional de las dietas}

Para evaluar la calidad nutricional de las dietas se realizaron análisis proximales en el Centro de Estudios en Ciencia y Tecnología de los Alimentos (CECTA) de la Universidad de Santiago. Los análisis se hicieron en concentrados de microalgas tradicionales y nativas cultivadas en botellas de $20 \mathrm{~L}$. En el caso de las dietas artificiales el jugo o concentrado nutritivo de Macrocystis 
pyrifera se obtuvo mediante el uso de un biodigestor. La diatomea utilizada para la formulación de la pasta fue la misma con que se alimentaron las larvas en el experimento con microalgas nativas rotulada como $\mathrm{ND}_{2}$.

\section{ANÁLISIS ESTADíSTICo}

Para los análisis estadísticos se utilizó el programa STATISTICA 8. Los datos de longitud de larvas se testearon para probar si cumplían los supuestos de normalidad y homogeneidad de varianzas. Para la variable longitud valvar se aplicó un test no paramétrico KruskalWallis y a posteriori el test de Mann-Whitney $(P<0,05)$. Para evaluar la supervivencia los datos fueron previamente transformados al arcoseno de la raíz del porcentaje. Posteriormente, se aplicó una ANOVA y luego una prueba de Tukey a posteriori $(P<0,05)$.

Se realizó un análisis de regresión para el crecimiento y supervivencia en el tiempo, seleccionándose un modelo exponencial. Los resultados obtenidos para las variables de supervivencia y crecimiento fueron linealizados y luego se realizó un análisis de covarianza (ANCOVA) cuya variable independiente correspondía a los tratamientos y una prueba de Tukey a posteriori. Se trabajó con un nivel de significancia de $P<0,05$ (Zar 1999).

Los análisis proximales de las dietas se analizaron en pares con una prueba $\mathrm{G}$ de independencia (Sokal \& Rohlf 2012).

\section{Resultados}

\section{Microalgas tradicionales}

El experimento con microalgas tradicionales mostró que es posible reconocer dos etapas en el crecimiento de las larvas. Una primera etapa se detecta entre el inicio del cultivo hasta el día 8 con una tasa diaria de crecimiento larval entre 5,0 y $6,3 \mu \mathrm{m} \mathrm{dia}{ }^{-1}$ para las diferentes dietas. La segunda etapa entre el día 10 y el término del cultivo larval mostró una tasa de crecimiento diario que duplica la etapa 1, con resultados entre $\operatorname{los} 10,8$ y $16,1 \mu \mathrm{m} \mathrm{dia}^{-1}$ (Tabla 1). En la etapa 1 , el mejor tratamiento con el que se obtienen la tasa de crecimiento y supervivencia más alta es con la dieta monoespecífica $I$. galbana $(I)$. En cambio en la etapa 2, el mejor tratamiento fue la dieta mixta $I / C c$ (Tabla 1 ).

La Figura 1 muestra el crecimiento y supervivencia de larvas de $M$. edulis alimentadas con las microalgas tradicionales $I, C c$ e $I / C c$. Durante los días 2, 4 y 6 del experimento las larvas alimentadas con $I$ presentaron un crecimiento mayor en sus 3 réplicas que los otros 2 tratamientos observándose diferencias significativas el día 8 de cultivo (Tabla 1). La longitud final fue de $272 \mu \mathrm{m}$ $( \pm 17,5 \mu \mathrm{m}$ ), con una tasa global de crecimiento (etapa 1+etapa2) de 9,2 $\mu \mathrm{m} \mathrm{día}^{-1}$ (Fig. 1A).

Las larvas alimentadas con $C c$ presentaron la menor tasa de crecimiento inicial para la etapa 1 , incrementándose a partir del día 10 (Tabla 1). La longitud final alcanzada por las larvas alimentadas con $C c$ fue de $272,2 \mu \mathrm{m}( \pm 25,4$

Tabla 1. Tasa de crecimiento diario (TCD) $\left(\mu \mathrm{m}\right.$ día $\left.^{-1}\right)$, longitud valvar $(\mu \mathrm{m})(M$ edia $\pm D$.E.) y supervivencia $(M$ edia $\pm D . E$.) de larvas de Mulinia edulis alimentadas con microalgas tradicionales, Isochrysis galbana (Clon T-Iso) (I), Chaetoceros calcitrans (CC) y el tratamiento mixto (I/CC). Para diferencias en longitud se utilizó una prueba de Kruskal-Wallis y una prueba de Mann-Whitney a posteriori, para supervivencia una prueba de ANOVA y para la tasa de crecimiento una ANCOVA y un test de Tukey a posteriori. Valores con letras diferentes en la misma columna indican que hay diferencias significativas $(\mathbf{P}<\mathbf{0 , 0 5})$ entre las dietas en cada etapa / Daily growth rate $(T C D)\left(\mu \mathrm{m}\right.$ day $\left.\mathbf{y}^{-1}\right)$ in valve length $(\mu \mathrm{m})(\mathrm{mean} \pm \mathrm{s} . \mathrm{d}$.) and survival (mean \pm s.d.) of larvae of Mulinia edulis fed with traditional microalgae Isochrysis galbana (Clone T-Iso) (I) and Chaetoceros calcitrans $(C C)$ alone and mixed $(I / C C)$. Differences in length were tested with the Mann-Whitney test, survival with ANOVA and growth rate with ANCOVA and an a posteriori Tukey test. Different letters in the same column indicate significant differences $(P<0.05)$ among the diets in each stage

\begin{tabular}{|c|c|c|c|c|}
\hline & Dietas & $\begin{array}{c}\text { TCD } \\
\left(\mu \mathrm{m} \mathrm{día}{ }^{-1}\right)\end{array}$ & $\begin{array}{c}\text { Longitud } \\
\text { (Media } \pm \text { D.E.) }\end{array}$ & $\begin{array}{c}\text { \% Supervivencia } \\
\text { (Media } \pm \text { D.E.) }\end{array}$ \\
\hline \multirow[t]{3}{*}{ Etapa 1} & $I$ & 6,6 (a) & $140,8 \pm 11,0(a)$ & $77,6 \pm 7,4$ (a) \\
\hline & C.c. & 5,0 (b) & $128,3 \pm 9,3$ (b) & $56,8 \pm 1,9(\mathrm{~b})$ \\
\hline & I/C.c. & 6,3 (a) & $138,3 \pm 10,9(\mathrm{c})$ & $75,7 \pm 1,5$ (a) \\
\hline \multirow[t]{3}{*}{ Etapa 2} & $I$ & $10,8(a)$ & $272,0 \pm 17,5$ (a) & $10,9 \pm 0,9$ (a) \\
\hline & C.c. & 11,9 (a) & $272,2 \pm 25,4$ (a) & $5,9 \pm 0,8(b)$ \\
\hline & I/C.c. & 16,1 (b) & $283,4 \pm 27,0(\mathrm{~b})$ & $13,6 \pm 0,7(\mathrm{c})$ \\
\hline
\end{tabular}



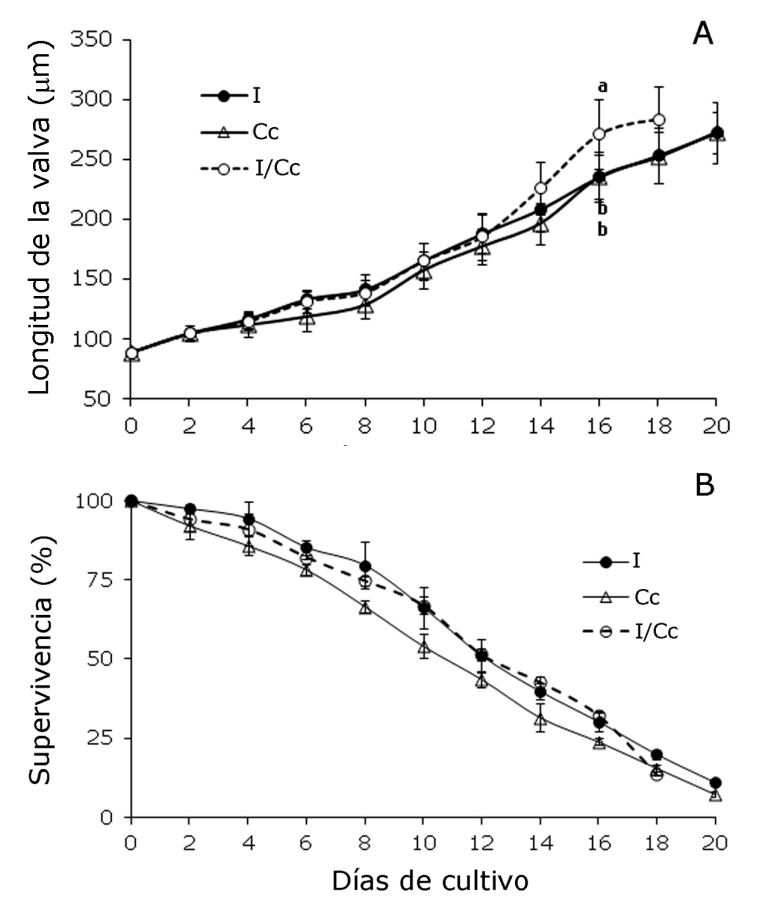

Figura 1. Crecimiento (A) y supervivencia (B) de larvas de Mulinia edulis alimentadas con microalgas tradicionales en los tratamientos monoespecíficos [Isochrysis galbana (Clon T-Iso) (I), Chaetoceros calcitrans (Cc)] y el tratamiento mixto (I/Cc) / Growth and survival of Mulinia edulis larvae fed with traditional microalgae in monospecific [(Isochrysis galbana (Clone T-Iso) (I), Chaetoceros calcitrans $(\mathrm{CC})]$ and mixed $(\mathrm{I} / \mathrm{CC})$ treatments

$\mu \mathrm{m})$, con una tasa de crecimiento global de $9,2 \mu \mathrm{m}$ día $^{-1}$ (Fig. 1A). Las larvas alimentadas con la mezcla $\mathrm{I} / \mathrm{Cc}$ presentaron un crecimiento lento hasta el día 8 de cultivo (Tabla 1), el que se incrementó a contar del día 10 alcanzando una longitud final de $283,4 \mu \mathrm{m}( \pm 26,9 \mu \mathrm{m})$. La tasa de crecimiento fue de $10,9 \mu \mathrm{m} \mathrm{día}^{-1}$ (Fig. 1A).

En la Figura 2 se muestra el ajuste exponencial al crecimiento de las larvas velígeras para los tres tratamientos con dietas tradicionales. El ANCOVA realizado al crecimiento valvar muestra diferencias significativas entre los tratamientos $(\mathrm{F}=118,63, P<0,001)$. La prueba de Tukey realizada a posteriori indica que existen diferencias significativas entre las combinaciones $I-C c \quad(P=0,00002)$, $I-I / C c(P=0,016)$ y $C c-I / C c(P=0,0007)$.

La supervivencia de las larvas tuvo un decrecimiento constante durante los 20 días de cultivo para los 3 tratamientos (Fig. 1B). La mortalidad de las larvas

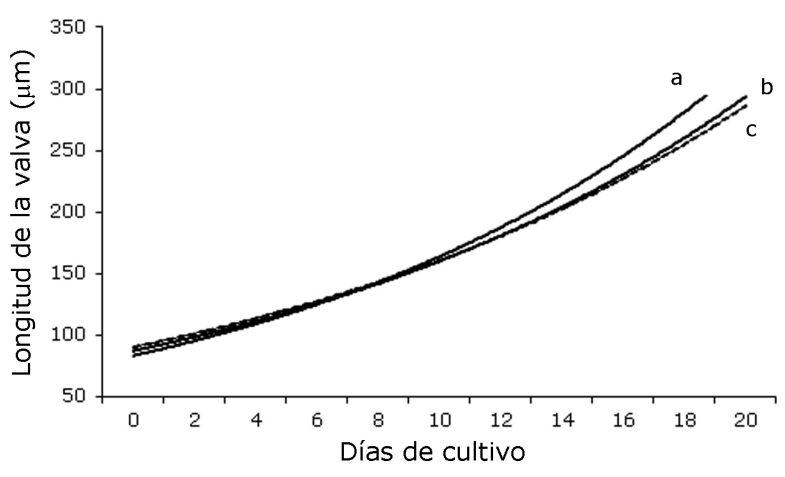

Figura 2. Modelo de crecimiento de larvas de Mulinia edulis alimentadas con microalgas tradicionales: a) tratamiento mixto Isochrysis galbana (Clon T-Iso) (I), Chaetoceros calcitrans (CC) I/CC $\left(r^{2}=0,95 ; y=81,695 e^{0,066 x}\right)$, b) tratamiento monoespecífico Cc $\left(r^{2}=\right.$ 0,$\left.96 ; y=82,573 e^{0,0578 x}\right)$ y tratamiento monoespecífico I $\left(r^{2}=0,95 ; y=\right.$ $\mathbf{8 6 , 8 7 3 e ^ { 0 , 0 5 6 7 x }}$ ) / Model of larval growth of Mulinia edulis fed with traditional algae: a) mixed treatment with Isochrysis galbana (Clone T-Iso) (I), Chaetoceros calcitrans (CC) I/CC $\left(r^{2}=0.95 ; y=\right.$ $\left.81.695 \mathrm{e}^{0.066 x}\right) ;$ b) monospecific treatment with $C c\left(r^{2}=0.96 ; y=\right.$ $\left.82.573 \mathrm{e}^{0.0578 x}\right)$ and $\mathrm{I}\left(\mathrm{r}^{2}=0.95 ; y=86.873 \mathrm{e}^{0.0567 x}\right)$

alimentadas con el tratamiento $I$ se incrementó a partir del día $12(50,9 \%)$. Para el día 20, día en que finalizó el experimento la supervivencia alcanzó el 10,9\%. Las larvas alimentadas con la diatomea $C c$ presentaron una disminución en la supervivencia a contar del día 4 de cultivo $(85,8 \%)$; esta característica se observó durante todo el experimento. La supervivencia final fue de 5,9\% y el experimento tuvo un tiempo de duración de 20 días.

El cultivo de larvas alimentadas con el tratamiento mixto $I / C c$ tuvo un tiempo de duración de 18 días y la supervivencia final fue de 13,6\% (Fig. 1B).

Un ANCOVA realizado a los datos de mortalidad mostró diferencias significativas $(\mathrm{F}=35,889 ; P<0,001)$. La prueba de Tukey realizada a posteriori indica que existen diferencias significativas entre las combinaciones $I / C c$ $(P=0,0001)$ y $C c-I / C c(P=0,0001)$ mientras que para la combinación $I-I / C c$ no se encontraron diferencias significativas $(P=0,07)$.

\section{Microalgas nativas}

La Figura 3A muestra el crecimiento en larvas de M. edulis alimentadas con microalgas nativas. Las larvas tuvieron una longitud inicial de $88,2 \mu \mathrm{m}( \pm 0,02 \mu \mathrm{m})$. Entre los días 0 al 2 de cultivo las larvas de todos los tratamientos presentan un longitud similar $(93,10-95,40 \mu \mathrm{m})$, sin 

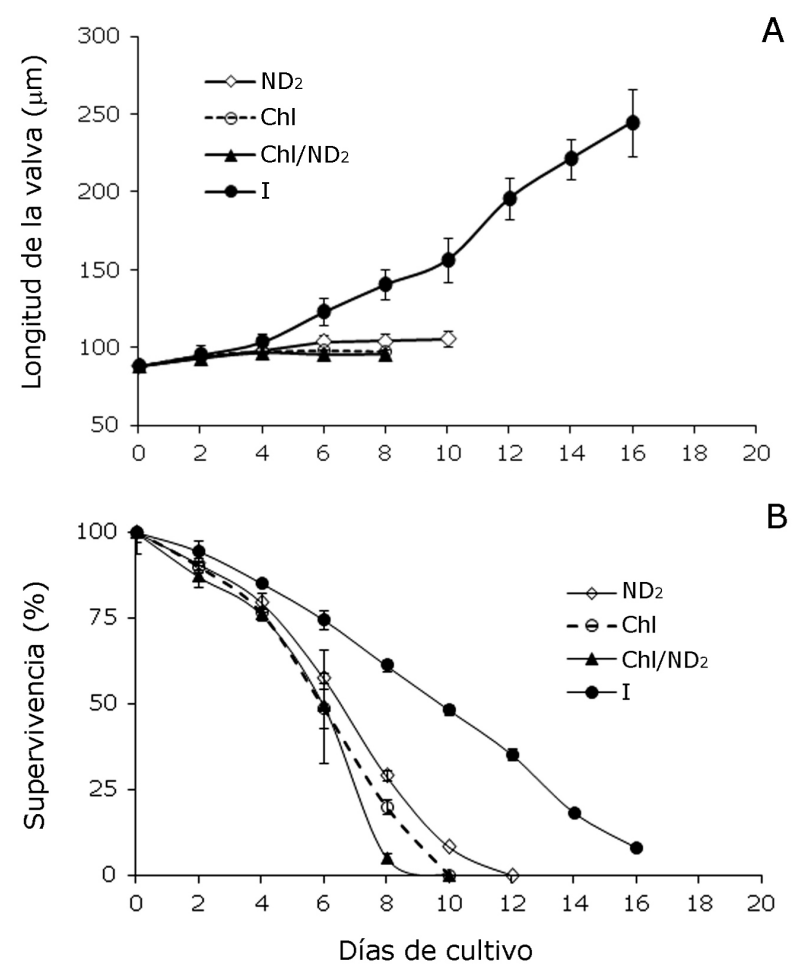

Figura 3. Crecimiento (A) y supervivencia (B) de larvas de Mulinia edulis alimentadas con microalgas nativas en tratamientos monoespecíficos [Chlamydomonas sp. (Chl), Diatomea Nativa $\left(\mathrm{ND}_{2}\right)$ ], tratamiento mixto $\left(\mathrm{Chl} / \mathrm{ND}_{2}\right)$ y como control Isochrysis galbana (Clon T-Iso) (I) / Growth and survival of Mulinia edulis larvae fed with native microalgae in monospecific [Chlamydomonas sp. (Chl), Native Diatom $\left.\left(\mathrm{ND}_{2}\right)\right]$ and mixed $\left(\mathrm{Chl} / \mathrm{ND}_{2}\right)$ treatments, using Isochrysis galbana (Clone T-Iso) (I) as control

embargo, a contar del día 4 comienzan a observarse diferencias. Las larvas de los tratamientos con $C h l / N D_{2}$ y Chl tuvieron una longevidad de 10 días en sus 3 réplicas y alcanzaron una longitud final de 95,6 $\mu \mathrm{m}( \pm 4,2 \mu \mathrm{m})$ y $97,46 \mu \mathrm{m}( \pm 3,9 \mu \mathrm{m})$, respectivamente, aquellas alimentadas con la diatomea $\mathrm{ND}_{2}$ tuvieron una longevidad de 12 días y una longitud final de $105,3 \mu \mathrm{m}( \pm 5,0)$, siendo eliminados los 3 tratamientos antes mencionados siguiendo solo el tratamiento control $(I)$. Las larvas en los tratamientos con microalgas nativas no alcanzaron la etapa de pedivelígeras. Las larvas alimentadas con la microalga control $(I)$ presentaron una longitud final de $244,8 \mu \mathrm{m}( \pm 21,5 \mu \mathrm{m})$ con una tasa de crecimiento de $6,7 \mu \mathrm{m}$ día $^{-1}$. El día 16 se puso fin al experimento debido a la presencia aproximada del $70 \%$ de larvas pedivelígeras en el cultivo.

Se observó una disminución en la supervivencia entre un 75,9 y $79,6 \%$ en las larvas alimentadas con microalgas nativas a partir del día 4 de cultivo (Fig. 3B). Durante el cambio de agua correspondiente al día 10 no se observaron larvas vivas en los tratamientos de Chl y Chl/ND 2. Las larvas alimentadas con la diatomea $\mathrm{ND}_{2}$ alcanzaron el $100 \%$ de mortalidad el día 12 de cultivo. Las larvas alimentadas con la microalga control $(I)$ alcanzaron una supervivencia final del 7,9\%.

\section{Dietas ARTIFICIALES}

La Figura 4A muestra el crecimiento en larvas de M. edulis alimentadas con dietas artificiales. Las larvas alimentadas con $\mathrm{CN}$ y CN/PD mantuvieron una longitud similar durante los primeros 8 días de cultivo, sin embargo, al día 12 de cultivo se decide terminar con estos tratamientos en sus 3 réplicas debido a que no se observaron larvas vivas en las muestras analizadas. Las longitudes finales fueron de $105,9 \pm 5,1 \mu \mathrm{m}$ y $105,2 \pm 6,0 \mu \mathrm{m}$, respectivamente.

En el caso de las larvas alimentadas con PD, la longitud final que alcanzaron las larvas fue de $114,6 \pm 7,3 \mu \mathrm{m}$ con una tasa de crecimiento de $1,3 \mu \mathrm{m}$ día $^{-1}$.

Las larvas alimentadas con la microalga control (I) presentaron un crecimiento mayor a las alimentadas con dietas artificiales a contar del día 4 de cultivo $(111,3 \mu \mathrm{m})$ alcanzando una longitud final de 276,8 $\pm 12,6 \mu \mathrm{m}$ con una tasa de crecimiento de $9,4 \mu \mathrm{m} \mathrm{día}{ }^{-1}$.

El ANCOVA realizado a los datos de longitud de las larvas muestra diferencias significativas $(\mathrm{F}=2272,2 ; P=$ $0,0001)$ entre aquellas alimentadas con la dieta PD y el grupo control (I).

La supervivencia tuvo una fuerte disminución en las larvas alimentadas con dietas artificiales a partir del segundo día de cultivo en adelante (Fig. 3B). Los tratamientos CN y CN/PD alcanzaron el $100 \%$ de mortalidad el día 14 de cultivo. La supervivencia final para las larvas alimentadas con el tratamiento PD fue de $0,31 \%$. Se decidió poner fin a este tratamiento en sus 3 réplicas el día 20 momento en que las larvas alimentadas con la microalga control (I) pasaron al estado pedivelíger.

La supervivencia de las larvas alimentadas con la microalga control (I) disminuyó de acuerdo avanzaban los días de cultivo. Para el día 20, día en que finalizó el experimento, la supervivencia alcanzó 5,62\%.

El ANCOVA realizado a los datos de mortalidad de las larvas mostraron diferencias significativas entre los tratamientos PD y el control $(I)(\mathrm{F}=12,247 ; P=0,002)$. 

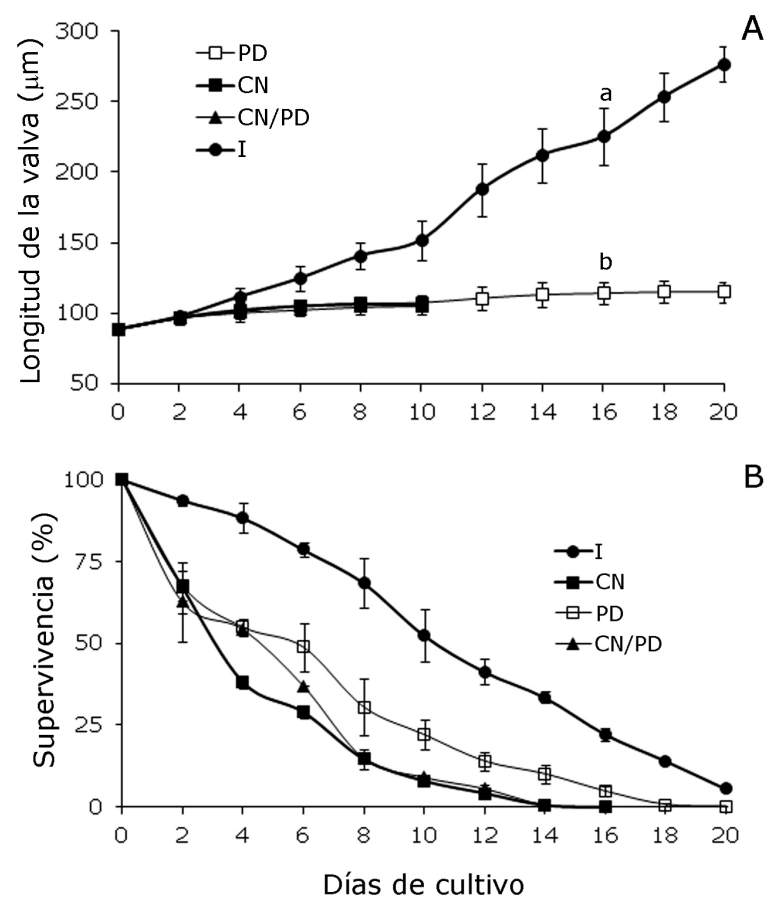

Figura 4. Crecimiento (A) y supervivencia (B) de larvas de Mulinia edulis alimentadas con las dietas artificiales en tratamientos monoespecíficos [Pasta de Diatomea (PD), Concentrado Nutritivo (CN)], tratamiento mixto (CN/PD) y como control Isochrysis galbana (Clon T-Iso) (I) / Growth and survival of Mulinia edulis larvae fed with artificial diet in monospecific [Diatom Paste (PD), Nutrient Concentrate (CN)] and mixed (CN/PD) treatments, using Isochrysis galbana (Clone T-Iso) (I) as contro
La composición nutricional de las dietas se describe en la Tabla 2. La composición proximal de las dietas son independientes entre sí con valores para $\mathrm{G}_{\text {adj }}$ entre 8,22 y 28,12 $(P<0,05)$, a excepción del PD cuya composición es idéntica a la microalga con que se preparó la dieta artificial. Las microalgas nativas Chlamydomona sp. (Chl) y la diatomea $\mathrm{ND}_{2}$ contienen los valores más altos de proteínas y lípidos, respectivamente. La microalga tradicional $C$. calcitrans y el concentrado nutritivo tienen los valores más altos para cenizas (materia orgánica) mientras que $I$. galbana (Clon T) el mayor valor para carbohidratos.

\section{Discusión}

Los resultados obtenidos con las microalgas tradicionales indica que el tratamiento mixto $I / C c$ genera el mejor resultado para las variables supervivencia y crecimiento en el cultivo de larvas de Mulinia edulis encontrándose diferencias significativas $(P<0,0001)$ en la tasa de crecimiento para los tratamientos monoespecíficos, lo que se explica principalmente por los valores proximales de ambas especies de microalgas. Este resultado concuerda con Galley et al. (2009) quienes indican que una dieta basada en una mezcla de microalgas es más rica en nutrientes que una monodieta. Durante los 4 primeros días de cultivo no se observaron diferencias significativas en la longitud valvar de las larvas velíger de la almeja taquilla, lo que es indicativo de que el crecimiento durante este periodo inicial depende de las reservas energéticas provenientes de la embriogénesis (Whyte et al. 1990).

Tabla 2. Composición proximal (\%) de las dietas suministradas a larvas velígeras de Mulinia edulis / Proximate composition (\%) of diets provided to veliger larvae of Mulinia edulis

\begin{tabular}{lcccc}
\hline \multicolumn{1}{c}{ Tratamiento } & Proteínas & Lípidos & $\begin{array}{c}\text { Carbohidratos } \\
\text { Totales }\end{array}$ & Cenizas \\
\hline Isochrysis galbana (I) & 32,8 & 15,5 & 25,9 & 25,9 \\
Chaetoceros calcitrans $($ Cc) & 31,3 & 10,8 & 10,8 & 47,3 \\
Chlamydomonas sp. (Chl) & 52,7 & 22,7 & 2,7 & 22,0 \\
Diatomea (ND $)$ & 36,0 & 27,2 & 9,6 & 27,2 \\
Concentrado Nutritivo (CN)* & 0,83 & 0,60 & nd & 99,5 \\
Pasta de Diatomea (PD) & 36,0 & 27,2 & 9,6 & 27,2 \\
\hline
\end{tabular}

* los valores proximales fueron determinados en base seca mediante su equivalencia en base húmeda 
Las diferencias observadas en los 3 tratamientos con microalgas tradicionales pudo deberse a que las larvas asimilaron en un principio la microalga flagelada Isochrysis galbana $(I)$ debido su fácil captura y características morfológicas a diferencia de Chaetoceros calcitrans $(C c)$, la que por su tamaño, falta de movilidad, pared celular y setas conformadas de silicio dificultan su ingestión y digestión, lo que explica las 2 etapas observadas (Tabla 1). Se debe tener en cuenta como señalan Cognie et al. (2001) que las larvas de bivalvos son capaces de elegir una microalga especifica dentro de una mezcla. Tang et al. (2006) describen que las larvas de la almeja Meretrix meretrix muestran preferencia por la microalga I. galbana dentro de una dieta mixta; este comportamiento alimentario podría ser relacionado a los nutrientes esenciales, morfología y tamaño de la microalga (Oliva et al. 2013).

El posterior aumento del tamaño de las larvas permite capturar células de $C c$ y la capacidad de digerir y nutrirse de esta microalga. Raghavan (2011) señala que en larvas de la almeja Veneridae, Paphia malabarica la tasa de filtración e ingestión aumenta de forma exponencial a medida que las larvas crecen.

Los requerimientos alimentarios para larvas de moluscos no están bien definidos por lo que cada especie requiere de una dieta particular lo que dificulta la comparación de los resultados obtenidos entre especies de un mismo grupo taxonómico (Knauer \& Southgate 1999). Las larvas de las almejas Meretrix meretrix (Tang et al. 2006) y Ruditapes philippinarum (Yan et al. 2006) ambas pertenecientes a la familia Veneridae alimentadas solo con Isochrysis galbana presentaron un mayor crecimiento que las alimentadas con tratamientos mixtos. Por el contrario, en larvas de la almeja Paphia malabarica también perteneciente a la familia Veneridae presentaron un crecimiento mayor al ser alimentadas con un tratamiento mixto basado en Isochrysis galbana y Nannochloropsis salina (Raghavan 2011).

Si bien los resultados no son comparables entre especies, estos resultados se asemejan a lo observado en la ostra Crassostrea gigas en que las larvas alimentadas con $C$. calcitrans tienen un mayor crecimiento pero una menor supervivencia que las alimentadas con I. galbana (Rico-Villa et al. 2006).

En el experimento con microalgas nativas, las larvas presentaron distintos resultados para cada tratamiento. Desde un comienzo se observó que las larvas alimentadas con $C h l$ presentaban una glándula digestiva transparente, lo que indica que no se estaban alimentando con esta microalga.

La dificultad en poder ingerir y digerir Chlamydomonas sp. pudo deberse a sus características morfológicas en la que destaca una pared celular de celulosa gruesa y rígida (Paniagua et al. 1986). Aldana-Aranda et al. (1997) alimentaron larvas velígeras del caracol Strombus gigas con Chlamydomonas coccoides presentando un bajo índice de ingestión en larvas de 1 día de cultivo, observándose la expulsión de partículas de alimento inicialmente ingeridas.

En el caso de la diatomea aislada del tracto digestivo $\left(N D_{2}\right)$ a pesar de haberse observado larvas con la glándula digestiva con un contenido moderado de alimento, la mortalidad larval llegó al 100\% explicándose en sus características morfológicas como es la falta de movimiento lo que dificulta su captura o su pared celular y setas formadas de sílice (Helm \& Bourne 2006). Knuckey et al. (2002) señalan que del sistema digestivo de juveniles de bivalvos se aíslan principalmente diatomeas y microalgas bentónicas y es en este estado en que se observan mejores resultados en crecimiento y supervivencia, lo que indicaría que la diatomea $\mathrm{ND}_{2}$ podría ser utilizada en semillas de la almeja obteniendo con mayores tasas de crecimiento y sobrevivencia.

En el tratamiento mixto $\mathrm{Chl} / \mathrm{ND}_{2}$ las larvas tuvieron un comportamiento similar a los otros 2 tratamientos monoespecíficos en los cuales se observa una glándula digestiva transparente, no presentan crecimiento y la mortalidad alcanza el $100 \%$ durante la mitad del periodo de cultivo.

Además de las características morfológicas de las microalgas nativas, otra posible causa de estos resultados radica en la calidad nutricional de éstas. A pesar de que Chlamydomonas sp. y la diatomea nativa $N D_{2}$ poseen una alta cantidad de lípidos y proteínas en comparación a I. galbana y C. calcitrans (Tabla 2), estas no aportarían los nutrientes necesarios, ya que no se obtuvieron las respuestas fisiológicas esperadas evidenciadas en el crecimiento y supervivencia.

En diversas especies de larvas de bivalvos se ha probado el efecto de microalgas aisladas desde el medio natural o del sistema digestivo. En larvas del pectínido Placopecten magellanicus (Gouda et al. 2006) las microalgas aisladas del medio natural generaron mejores resultados en las variables crecimiento y supervivencia que las microalgas utilizadas comúnmente en acuicultura. Mientras que en larvas del pectínido Argopecten 
purpuratus alimentadas con diatomeas aisladas de la columna de agua no se encontraron diferencias significativas en el crecimiento utilizando distintas especies de diatomeas (Carvajal et al. 2007) ${ }^{1}$.

En el experimento realizado con dietas artificiales se encontraron diferencias significativas $(P<0,0001)$ para las tasas de crecimiento y mortalidad entre el tratamiento control $I$ y PD. Por las características morfológicas o la manera en que fueron suministrados los tratamientos PD y $\mathrm{CN}$, las larvas no fueron capaces de asimilar éstos evidenciando características de inanición con una glándula digestiva transparente, a pesar de esto, las larvas vivas presentaban natación durante el periodo que duró su cultivo, similar a larvas de Ruditapes decussatus capaces de estar 23 días sin recibir alimento (Matias et al. 2011).

El resultado obtenido alimentando larvas con PD pudo deberse a las dificultad presentada en el experimento para separar las células, concordando con Heasman et al. (2000) en donde las partículas de la pasta superan el tamaño apto para que las larvas se alimenten.

En estudios con otras especies se han obtenido diversos resultados; en el caso de larvas de la ostra Crassostrea gigas alimentadas con concentrados de $C$. calcitrans generados por floculación, el resultado fue exitoso en las variables crecimiento y supervivencia suministrada de manera total $(100 \%)$ o de forma parcial (siendo acompañada por una microalga viva) (Brown \& Robert 2002). Haesman et al. (2000) demostraron que concentrados de microalgas con una apropiada preservación y almacenamiento son capaces de reemplazar a las microalgas frescas en dietas de larvas de bivalvos (ostras y ostiones).

Helm \& Bourne (2006) señalan que el medio nutritivo en que se cultivan las microalgas es capaz de nutrir larvas de 1 día de vida y que aún no tienen el tamaño suficiente para alimentarse de la microalga. Manahan (1983) señala que larvas de los bivalvos Crassotreas gigas y Mytilus edulis son capaces de absorber nutrientes disueltos desde el agua de mar en un sistema de cultivo supliendo la carencia de alimento cuando las reservas provenientes de la embriogénesis disminuyen.

La utilización del biodigestor genera buenos resultados para la fabricación de un medio de cultivo para microalgas (Paniagua \& Bückle 1985), sin embargo, para el caso de las larvas alimentadas con el CN la falta de los nutrientes necesarios para ayudar al crecimiento o la nula capacidad de asimilación pudo provocar que la mortalidad llegara al $100 \%$ durante los primeros días de cultivo. Por otra parte, la característica del CN como su viscosidad, pudo impedir una fácil digestión y movimiento de los cilios.

Se han generado distintas metodologías para el uso de macroalgas como dieta para bivalvos. Pérez-Camacho et al. (2004) produjeron a partir de Laminaria saccharina detritus de la macroalga utilizando bacterias y enzimas (Single Cell Detritus) como resultado solo se observó un efecto positivo en semillas de la almeja Ruditapes decussatus aumentando su peso y tamaño, sin embargo al usarlas en larvas de la misma especie se alcanzó una mortalidad del $100 \%$ hacia el día 10 de cultivo larval. Únicamente Uchida \& Numaguchi (1996) han descrito cómo las larvas de almeja japonesa $R$. philippinarum son capaces de ingerir detritos obtenidos a partir de la degradación del alga Ulva pertusa por bacterias marinas.

Los resultados obtenidos de las 3 dietas puestas a prueba indican que los mejores resultados se obtienen con dietas basadas en microalgas tradicionales y de estas, el tratamiento mixto compuesto por I. galbana y $C$. calcitrans entrega la mayor supervivencia y mayor tasa de crecimiento. Estas microalgas además, aportan los nutrientes necesarios como lípidos y proteínas. Matias et al. (2011) señalan que en la almeja $R$. decussatus lípidos y proteínas son la principal fuente de energía en el desarrollo larval.

Sin embargo, el siguiente paso es suministrar $I$. galbana y $C$. calcitrans en 2 etapas, como lo demuestran los resultados del experimento con microalgas tradicionales. Primero desde el inicio (día 0) hasta el día 8 alimentar sólo con I. galbana y desde el día 9 proporcionar una dieta mixta con $C$. calcitrans hasta la aparición de larvas pedivelígeras.

Ante la dificultad aún de generar una metodología en la que las larvas sean capaces de ingerir ya sea de manera sólida (detritus) o líquida (jugo o concentrados nutritivos) los estudios se han enfocado principalmente en semillas de bivalvos (Albentosa \& Pérez-Camacho 2002, PérezCamacho et al. 2004). Por lo tanto es necesario probar el efecto que puedan tener las microalgas nativas en semillas de $M$. edulis y a la vez profundizar en la calidad nutricional que estas pueden proporcionar.

${ }^{1}$ Carvajal CA, A Araya, E Pérez, A Briceño, R Hernández \& M Madrigal. 2007. Utilización de microalgas aisladas del medio natural para el enriquecimiento de dietas para larvas de ostión del norte (Argopecten purpuratus). 1er Congreso Nacional de Acuicultura, Coquimbo, 355 pp. 
Aunque los experimentos se realizaron de manera secuencial, este estudio proporciona la información necesaria para generar un protocolo para la alimentación de larvas de M. edulis en un sistema de cultivo.

\section{Agradecimientos}

Los autores agradecen a Gloria Collantes y Alfredo Astorga por su ayuda en la aislación y mantención de las microalgas nativas, a Gonzalo Fernandez de Orizon S.A. por su permanente apoyo, a Ángela Celis por su colaboración logística en el hatchery, a Mauricio Landaeta en los análisis estadísticos y a L. René Durán y los revisores anónimos por sus comentarios para enriquecer este manuscrito. Esta investigación es parte de la tesis para obtener el título de biólogo marino de Giovanni Vivanco y se realizó durante la ejecución del proyecto FONDEF/HUAM AQ08I1027.

\section{LITERATURA CITADA}

Abarca A, D Oliva, R Gutiérrez, A Celis \& LR Durán. 2012. Grown-out of seeds of the taquilla clam Mulinia edulis (King \& Broderip, 1832) in the subtidal zone in northern Chile and in the intertidal zone in southern Chile. Latin American Journal of Aquatic Research 40(3): 694-704.

Albentosa M \& A Pérez-Camacho. 2002. Valor nutritivo de harinas de macroalgas para el cultivo de semilla de almeja japonesa Ruditapes philippinarum (Adams \& Reeve, 1850): pruebas preliminares. Boletín del Instituto Español de Oceanografía 18(1-4): 281-287.

Aldana-Aranda D, V Patiño-Sukez \& T Brulé. 1997. Nutritional potentialities of Chlamydomonas coccoides and Thalassiosira fluviatilis, as measured by their ingestion and digestion rates by the Queen Conch larvae (Strombus gigas). Aquaculture 156: 9-20.

Brown MR. 2002. Nutritional value and use of microalgae in aquaculture. In: Cruz-Suárez LE, D Ricque-Marie, M TapiaSalazar, MG Gaxiola-Cortés \& N Simoes (eds). Avances en Nutrición Acuícola VI. Memorias del VI Simposium Internacional de Nutrición Acuícola, Cancún, pp. 281-292.

Brown MR \& R Robert. 2002. Preparation and assessment of microalgal concentrates as feeds for larval and juvenile Pacific oyster (Crassostrea gigas). Aquaculture 207: 289309.

Cognie B, L Barillé \& $\mathbf{E}$ Rincé. 2001. Selective feeding of the oyster Crassostrea gigas fed on a natural microphytobenthos assemblage. Estuaries 24: 126-134.

Collantes G \& D Oliva. En prensa. Métodos de cultivo de microalgas, 146 pp. Editorial Universidad de Valparaíso, Valparaíso.
Cruz-Suárez LE, D Ricque-Marie, M Tapia-Salazar \& C Guajardo-Barbosa. 2000. Uso de harina de kelp (Macrocystis pyrifera) en alimentos para camarón. En: CruzSuarez LE, D Ricque-Marie, M Tapia-Salazar, MA OlveraNovoa \& E Civera-Cerecedo (eds). Avances en Nutrición Acuícola V. Memorias del V Simposium Internacional de Nutrición Acuícola, Yucatán, pp. 19-22.

Duerr E, A Molnar \& V Sato. 1998. Cultured microalgae as aquaculture feeds. Journal of Marine Biotechnology 7: 6570.

Galley TH, FM Batista, R Braithwaite, J King \& AR Beaumont. 2009. Optimisation of larval culture of the mussel Mytilus edulis (L.). Aquaculture International 18: 315-325.

Gouda R, E Kenchington, B Hatcher \& B Vercaemer. 2006. Effects of locally-isolated micro-phytoplankton diets on growth and survival of sea scallop (Placopecten magellanicus) larvae. Aquaculture 259: 169-180.

Heasman MP, J Diemar, W O'Connor, T Sushames \& L Foulkes. 2000. Development of extended shelf-life microalgae concentrate diets harvested by centrifugation for bivalve mollusks- a summary. Aquaculture Research 31: 637-659.

Helm M \& N Bourne. 2006. Cultivo de bivalvos en criadero. Un manual práctico. FAO Documento Técnico de Pesca 471: 1-198.

Hemaiswarya S, R Raja, RR Kumar, V Ganesan \& C Anbazhagan. 2011. Microalgae: a sustainable feed source for aquaculture. World Journal of Microbiology \& Biotechnology 27: 1737-1746.

Knauer J \& P Southgate. 1999. A review of the nutritional requirements of bivalves and the development of alternative and artificial diets for bivalve aquaculture. Reviews in Fisheries Science 7: 241-280.

Knuckey RM, MR Brown, SM Barrett \& GM Hallegraeff. 2002. Isolation of new nanoplanktonic diatom strains and their evaluation as diets for juvenile Pacific oyster (Crassostrea gigas). Aquaculture 211: 253-274.

Lavens P \& P Sorgeloos. 1996. Manual on the production and use of live food for aquaculture. FAO Fisheries Technical Paper 361: 1-305.

Manahan D. 1983. The uptake and metabolism of dissolved amino acids by bivalve larvae. The Biological Bulletin 164: 236-250.

Marshall R, S Mckinley \& C Pearce. 2010. Effects of nutrition on larval growth and survival in bivalves. Reviews in Aquaculture 2(1): 33-55.

Matias D, S Joaquim, M Ramos, P Sobral \& A Leitao. 2011. Biochemical compounds' dynamics during larval development of the carpet-shell clam Ruditapes decussatus (Linnaeus, 1758): effects of mono-specific diets and starvation. Helgoland Marine Research 65: 369-379. 
O'Connor W \& MP Heasman. 1997. Diet and feeding regimens for larval doughboy scallops, Mimachlamys asperrima. Aquaculture 158: 289-303.

Oliva D, A Cifuentes, A Abarca, R Farlora, P Vera, LR Durán, J Urra, A Urra, D Brown, A Celis \& R Gutiérrez. 2005. Cultivo comercial de la almeja fina Mulinia edulis, 45 pp. Universidad de Valparaíso, Valparaíso.

Oliva D, A Abarca, R Gutiérrez, Á Célis, L Herrera \& V Pizarro. 2013. Effect of stocking density and diet on growth and survival of post-larvae of the taquilla clam Mulinia edulis cultivated in sand in a hatchery. Revista de Biología Marina y Oceanografía 48(1): 37-44.

Paniagua J \& L Bückle. 1985. Cultivo en condiciones controladas de Monochrysis lutheri y Skeletonema costatum con extractos de macrófitas marinas (fitoplancton). Anales del Instituto Ciencias del Mar y Limnología, Universidad Nacional Autónoma de México 12(1): 59-70.

Paniagua J, F Bückle, C Granados \& D Loya. 1986. Manual de metodologías y alternativas para el cultivo de microalgas, 94 pp. CICESE, Ensenada.

Pérez-Camacho A, JM Salinas, C Fuertes \& M Delgado. 2004. Preparation of single cell detritus from Laminaria saccharina as a hatchery diet for bivalve mollusks. Marine Biotechnology 6: 642-649.

Ponis E, I Probert, B Veron, M Mathieu \& R Robert. 2006. New microalgae for the Pacific oyster Crassostrea gigas larvae. Aquaculture 253: 618-627.

Raghavan G. 2011. Influence of algal cell size on filtration and ingestion rates during different larval stages of yellow neck clam, Paphia malabarica Chemnitz. Aquaculture Nutrition 37(3): 327-331.

Rico-Villa B, JR Le Coza, C Minganta \& R Robert. 2006. Influence of phytoplankton diet mixtures on microalgae consumption, larval development and settlement of the Pacific oyster Crassostrea gigas (Thunberg). Aquaculture 256(1-4): 377-388.

Rivero-Rodríguez S, AR Beaumont \& MC Lora-Vilchis. 2007. The effect of microalgal diets on growth, biochemical composition, and fatty acid profile of Crassostrea corteziensis (Hertlein) juveniles. Aquaculture 263: 199-210.
Sargent JR, LA McEvoy \& JG Bell. 1997. Requirements, presentation and sources of polyunsaturated fatty acids in marine fish larval feeds. Aquaculture 155: 117-127.

SERNAPESCA. 2011. Anuario estadístico de pesca. Servicio Nacional de Pesca, Valparaíso. <http://www.sernapesca.cl/ index.php?option=com_remository\&Itemid $=54 \&$ func $=$ select\&id $=2>$

Sokal RR \& FJ Rohlf. 2012. Biometry. The principles and practice of statistics in Biological Research, 937 pp. W.H. Freeman and Company, New York.

Spolaore P, C Joannis-Cassan, E Duran \& A Isambert. 2006. Review. Commercial Applications of Microalgae. Journal of Bioscience and Bioengineering 101(2): 87-96.

Tang BJ, BZ Liu, GD Wang, T Zhang \& JH Xiang. 2006. Effects of various algal diets and starvation on larval growth and survival of Meretrix meretrix. Aquaculture 254: 526533.

Uchida M \& K Numaguchi. 1996. Formation of protoplasmic detritus with characteristics favorable as food for secondary animals during microbial decomposition of Ulva pertusa (Chlorophyta) frond. Journal of Marine Biotechnology 4: 200-206.

Walne PR. 1974. Culture of bivalve molluscs. 50 years experience at Conway, 189 pp. Fishing News Books, London.

Wendt D \& C Johnson. 2006. Using latent effects to determine the ecological importance of dissolved organic matter to marine invertebrates. Integrative and Comparative Biology 46(5): 634-642.

Whyte J, N Bourne \& N Ginther. 1990. Biochemical and energy changes during embryogenesis in the rock scallop Crassodoma gigantean. Marine Biology 106: 239-244.

Yan XW, GF Zhang \& F Yang. 2006. Effects of diet, stocking density, and environmental factors on growth, survival, and metamorphosis of Manila clam Ruditapes philippinarum larvae. Aquaculture 253: 350-358.

Zar JH. 1999. Biostatistical analysis, 660 pp. Prentice Hall, Englewood Cliffs. 